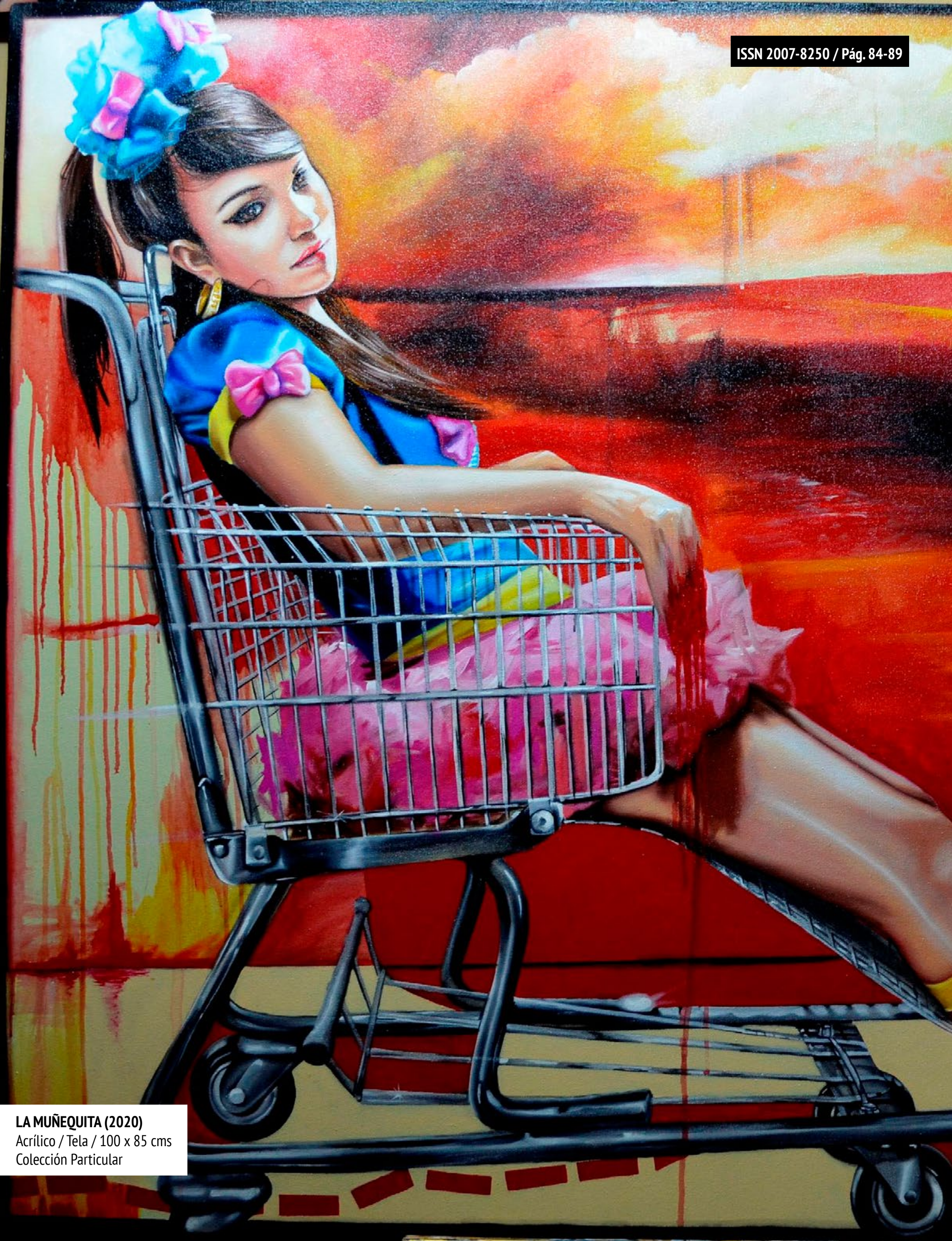


 \\ EL IMPACTO ACADÉMICO
}

\section{DEL PROGRAMA MUNDO DE LOS MATERIALES EN ALUMNOS DE LA PREPARATORIA 8}

THE ACADEMIC IMPACT OF THE MWT PROGRAM IN PREPARATORIA 8

\author{
LUCY MAYELA OCHOA RODRÍGUEZ \\ https://orcid.org/0000-0001-8424-7905 \\ BLANCA ELIZABETH GARZA GARZA \\ https://orcid.org/0000-0003-0752-351X
}

RESUMEN

El presente estudio busca conocer el impacto en los promedios de los alumnos de la Preparatoria 8 que participan en el Programa de Mundo de Materiales e identificar su participación por sexo en las diversas áreas de intereses, antes y después de la aplicación del programa. Los participantes del estudio fueron 83 alumnos que se inscribieron, $66 \%$ femenino y $34 \%$ masculino, de tercer semestre de bachillerato general, con una edad entre 16 y 17 años. Se contó con el apoyo de dos profesores, un coordinador y un coordinador general. En los cambios de intereses se reflejaron en un $30 \%$ de los participantes, estos a su vez hicieron el cambio de área de $81 \%$ al área de Ciencia y Tecnología. La modificación de su promedio se reflejó de un 75 a un 78, con avance de tres puntos, siendo las mujeres las que avanzaron más este apartado. Se concluye que hay un impacto en el promedio debido a la participación de programas extra aula.

PALABRAS CLAVE:

Materiales, Impacto, Alumnos, Académico, Bachillerato, Programa
ABSTRACT

This study seeks to know the impact on the averages of High School 8 students who participate in the World of Materials Program, as well as to identify the participation of students by gender in the various areas of interest, before and after the application of the program. The study participants were 83 students who enrolled, $66 \%$ female and $34 \%$ male, from the third semester of general high school, with an age between 16 and 17 years. It had the support of two teachers, a coordinator and a general coordinator. In the changes of interest, they were reflected in $30 \%$ of the participants, these in turn made the change of area from $81 \%$ to the area of Science and Technology. The modification of their average was reflected from 75 to 78 , with an advance of three points, with women who advanced the most in this section. It is concluded that there is an impact on the average due to the participation of extra-classroom programs.

KEYWORDS:

Materials, Impact, Students, Academic, Baccalaureate, Program 
E I Mundo de los Materiales (MWM) es un programa educativo diseñado para complementar los cursos tradicionales de ciencia, matemáticas y tecnología para estudiantes de preparatoria. Inició en 1993 con el apoyo de la Northwestern University (USA) y una beca de la Fundación Nacional para la Ciencia (NSF), posteriormente fue desarrollado por la Iniciativa $\mathrm{Na}$ notecnológica Nacional y el Departamento de Defensa e Industria (USA) (Martínez-Sánchez, 2010).

La prioridad principal del programa fue el diseño de actividades que promovieron el aprendizaje cooperativo y la investigación científica mediante una serie de módulos interdisciplinarios basados en temas específicos de la ciencia de los materiales (Fuentes Cobas L, 2011).

Cada módulo contiene una serie de actividades prácticas que inician con una actividad introductoria que despierta el interés de los estudiantes en el tema, posteriormente cuatro o cinco actividades que facilita a los estudiantes la comprensión básica de los principios y conceptos que forman parte de un proyecto y finalizan con el diseño de un prototipo óptimo y funcional (Pérez, 2021).

En la Universidad Autónoma de Nuevo León, los alumnos de nivel medio superior cada semestre son invitados a participar sin algún costo en actividades prácticas complementarias para su formación académica, estas prácticas son diseñadas para mejorar y enriquecer el plan de estudio. Lo principal de estas actividades es fomentar la participación; sin embargo, hay muy poco interés en participar.

Se les invita a formar parte de los programas extraacadémicos, como una manera de apoyar el desarrollo de sus competencias en ese periodo, que pueden ser desde una serie de actividades por instructores internos o externos, también se lleva a cabo, una serie de propaganda e invitación en línea y presencial para formar parte de los diferentes programas que se imparten. En ocasiones se llega a convenir la participación con incentivos de carácter académico y, aun así, hay poca asistencia.

La forma en la cual se abordará esta problemática es mostrando los resul-
La prioridad principal del programa

fue el diseño de actividades que promovieron

el aprendizaje cooperativo y la investigación

científica. (Fuentes Cobas L. 2011) tados del impacto académico en estudiantes que participan en el Programa del Mundo de Materiales, sobre todo en unidades de aprendizaje relacionadas con la ciencia y tecnología, así como también el aumento por el interés en carreras universitarias con el mismo perfil (Fuentes Cobas L., 2011).

Por tal motivo, se ha intensificado el seguimiento de la aplicación del Programa MM, especificando los intereses antes y después de la aplicación, en los alumnos; así como sus referencias académicas.

De este planteamiento surge la presente investigación, la cual parte de los siguientes objetivos:

- Describir la distribución de los cambios en los intereses por áreas, antes y después de participar en el programa.

- Presentar los resultados de las calificaciones promedio de los participantes en el programa.

\section{JUSTIFICACIÓN}

\section{DE LA INVESTIGACIÓN}

El programa tiene como objetivo incrementar el ingreso de estudiantes al área de ciencia y tecnología. Lo anterior con la utilización de nuevas metodologías de enseñanzas e investigación que implica una mejor comprensión en diferentes temas relacionados con la Física, Química, Biología - Matemática, a través del conocimiento de la ciencia y tecnología de los materiales.

Las actividades diseñadas en cada módulo ofrecen a los estudiantes la oportunidad de involucrarse en procesos relacionados con la ciencia de los materiales en torno a problemas de ingeniería del mundo real. Esto 
permite que el método diseñado de investigación apoye a entender mejor la ciencia.

\section{OBJETIVO GENERAL}

Conocer el impacto académico en los alumnos participantes de la Preparatoria 8 en el Programa del Mundo de Materiales en el periodo del 2019.

\section{OBJETIVO ESPECÍFICO \\ DE LA INVESTIGACIÓN}

Identificar la participación de los alumnos por sexo y edad que estuvieron en el Programa del Mundo de Materiales en el 2019.

\section{MÉTODO}

- Selección de la Muestra (descripción). Se establecieron los resultados del impacto académico con los 83 participantes, a través de la obtención de los promedios totales de las calificaciones del semestre cursado en el 2019.

- Instrumento de Investigación. Pre-test y pos-test de cada uno de los módulos del programa.

- Procedimiento. Se aplican encuestas de entrada y de salida en cada uno de los módulos a los participantes.

\section{HIPÓTESIS}

Con lo anterior, el programa Mundo de los Materiales busca transformar la educación científica en el Nivel Medio Superior con el propósito de incrementar el ingreso de estudiantes en las áreas médica, de ciencia y tecnología, esto con el apoyo de un método diferente de enseñanza que implica mayor comprensión en temas de las materias de Física, Química, Biología o Matemáticas a través del conocimiento de la ciencia y tecnología de los materiales.

\section{Se requiere de la implementación} de estrategias que fortalezcan las competencias del profesor en las áreas que constituyen los módulos de Mundo de los Materiales para beneficio de los estudiantes y del propio programa.

\section{RESULTADOS}

Los resultados a esta fecha fueron que el $30 \%$ de los estudiantes incrementaron su promedio en el período agosto - diciembre 2018, mientras que el $15.34 \%$ de los estudiantes lo mantuvo de un período a otro, dando un total de $45.34 \%$ de estudiantes que reforzaron y/o consolidaron sus competencias en función de la mejora en su promedio académico después de haber cursado el programa. Asimismo, el análisis también muestra que el $78.33 \%$ de los estudiantes mantuvieron su promedio en un rango de calificación de 80 a 100 puntos.

\section{CONCLUSIONES}

Al inicio de bachillerato los estudiantes no tienen aún una definición clara respecto a su proyecto de vida, más aún la profesión que elegirán y de la cual iniciarán su misión y visión de vida. Es por eso que es necesario que el Sistema Educativo, en particular, el Nivel Medio Superior, tienda hacia una formación integral, de tal forma que le permita conocer al estudiante los espacios de acción de las diversas disciplinas que cursan a través de su ciclo de bachillerato de manera que pueda descubrir sus habilidades, aptitudes y conocimientos, para iniciar con una elección profesional apropiada.

En este sentido, la implementación de proyectos como el Mundo de los Materiales puede estimular a los estudiantes de bachillerato a sentir un mayor interés por la ciencia. Eso les puede motivar a que en un futuro cercano opten por una carrera cuyos programas educativos comprendan la asimilación de conceptos relacionados con la Ciencia, la Tecnología, la Ingeniería y las Matemáticas.

Parte fundamental del programa lo constituye la participación del personal docente. Los profesores son conscientes de que cuando se promueve la participación y se utilizan distintos recursos y técnicas innovadoras, por lo general se consiguen buenos resultados porque cambia la actitud y el compromiso de los estudiantes.

Por lo anterior, se requiere de la implementación de estrategias que fortalezcan las competencias del profesor en las áreas que constituyen los módulos de Mundo de los Materiales para beneficio de los estudiantes y del propio programa. 


\section{REFERENCIAS}

Fuentes Cobas, D. (2011). Proyectos exitosos financiados por Fondo Mixto CONACYT. Centro de Investigación en Materiales Avanzados, S.C., s/n.

Fuentes Cobas, L. (14 de noviembre de 2019). Programa MWM-México. Obtenido de El Mundo de los Materiales en México: http://mwm.cimav.edu.mx/

Pérez, Antonio (febrero de 2021). Prácticas del Mundo de Materiales. Obtenido de El Mundo de los Materiales en México: http://mwm.cimav.edu.mx/escue$\underline{\text { la-2021/ }}$

Martínez-Sánchez, R. (2010). Versión para América Latina del Programa "Módulos: El Mundo de los Materiales! (Materials World Modules). La educación. Revista Digital (144).

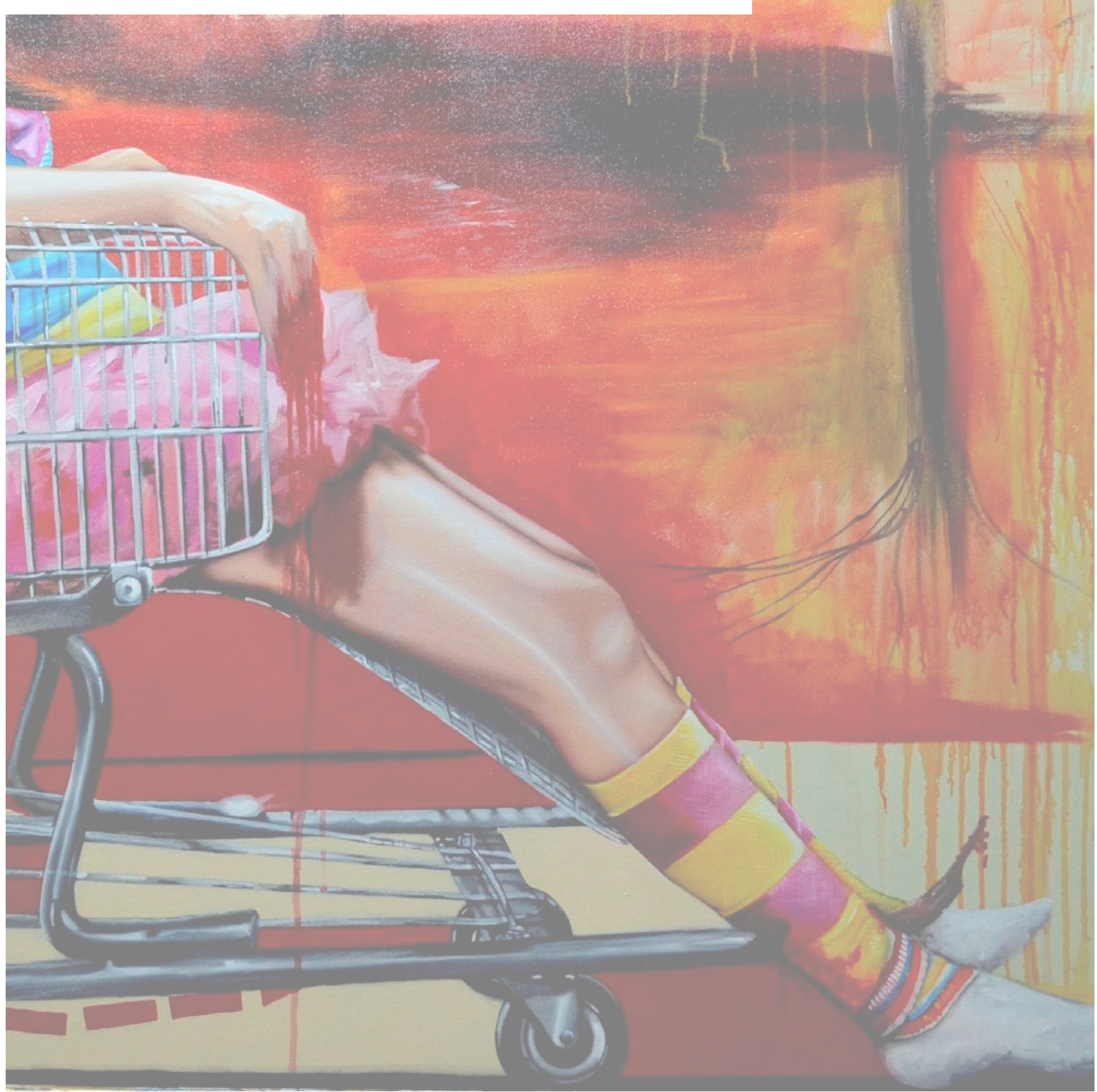




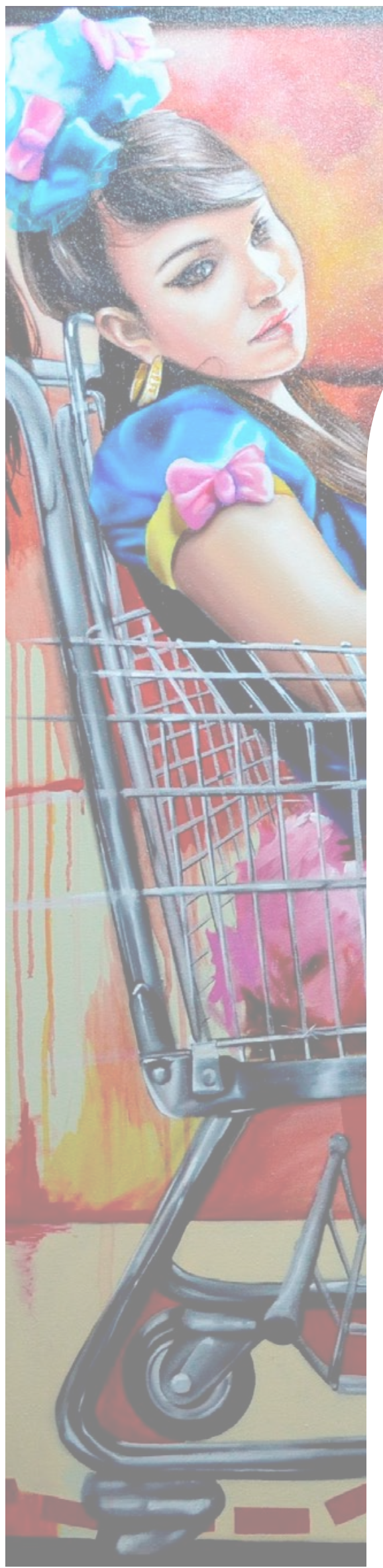

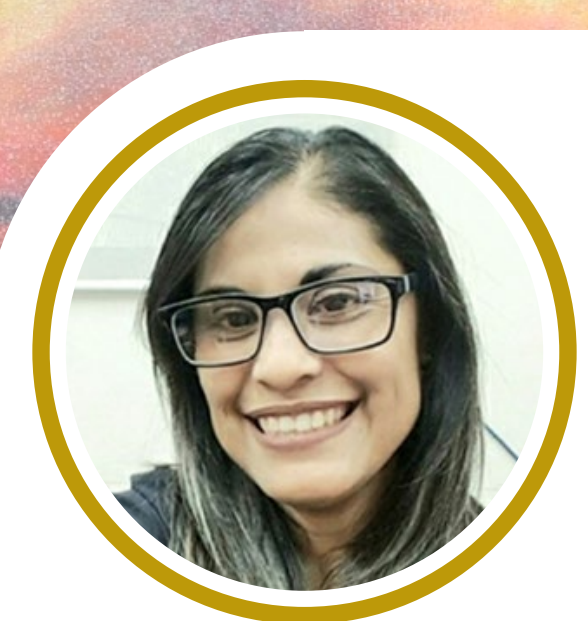

\section{LUCY MAYELA OCHOA RODRÍGUEZ}

Es Licenciada en Organización Deportiva por la FOD y egresada de la Maestría en Educación con Orientación en Didáctica Constructivista. Su experiencia en la docencia la ha desarrollado en distintos niveles educativos impartiendo cátedras de ciencias y de humanidades. Es colaboradora activa del programa "Mujeres en la ciencia" de la UANL. Se desempeña actualmente como Jefe de Laboratorios de Ciencias así también como Coordinadora del taller "El mundo de los materiales" y Docente de tiempo completo en la Preparatoria 8 de la UANL.

Correo Electrónico: carnomi@hotmail.com

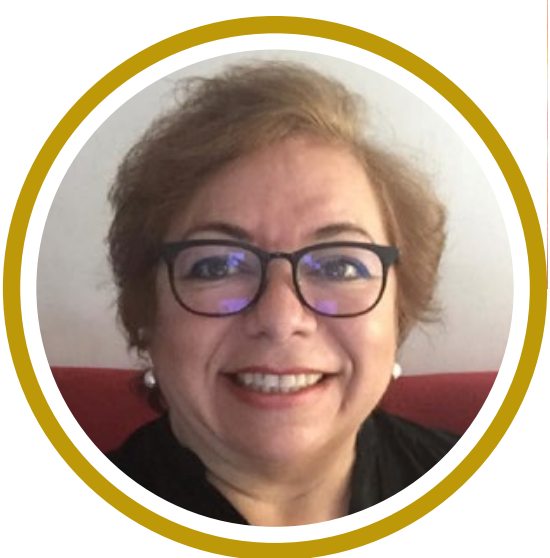

\section{BLANCA ELIZABETH GARZA GARZA}

Es Licenciada en Psicología con acentuación en área laboral y egresada de la Maestría en Ciencia en Orientación Vocacional por la Facultad de Psicología de la Universidad Autónoma de Nuevo León y la Universidad de Göttingen en Alemania. Se ha desempeñado como Coordinador Académico y encargada del Departamento de Orientación, así como Directora en la Universidad TecMilenio. Actualmente es Representante de los Cuerpos Académicos de Desarrollo Humano en la Dirección de Educación del Sistema de Nivel Medio Superior en la UANL y Profesor Docente en la Preparatoria 8.

Correo Electrónico: bettzy13@hotmail.com 


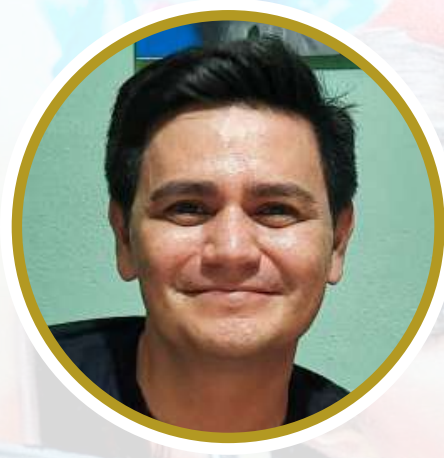

\section{Carlos Enrique Robledo Moreno, 1981, Monterrey, Nuevo León}

Artista egresado de la Facultad de Artes Visuales de la Universidad Autónoma de Nuevo León. La mayor parte de su obra la realiza en murales por las principales avenidas del municipio de San Nicolás de los Garza, Nuevo León, México. Coordina el programa de Arte Urbano en el Instituto Municipal para el Desarrollo Cultural (IMDEC) de San Nicolás de los Garza. Además, realiza su obra en lienzo y en el arte Madonnari (gis/pavimento). Ha expuesto y pintado de manera local, nacional e internacionalmente en paises como Francia, Italia y Estados Unidos. Ha recibido importantes reconocimientos como en Nocera Superiore, Italia, 2013 (2do. lugar del público) y 2015 (1er lugar del público); y en Wilhelmshaven, Alemania, 2019 (3er Lugar Categoría Libre).

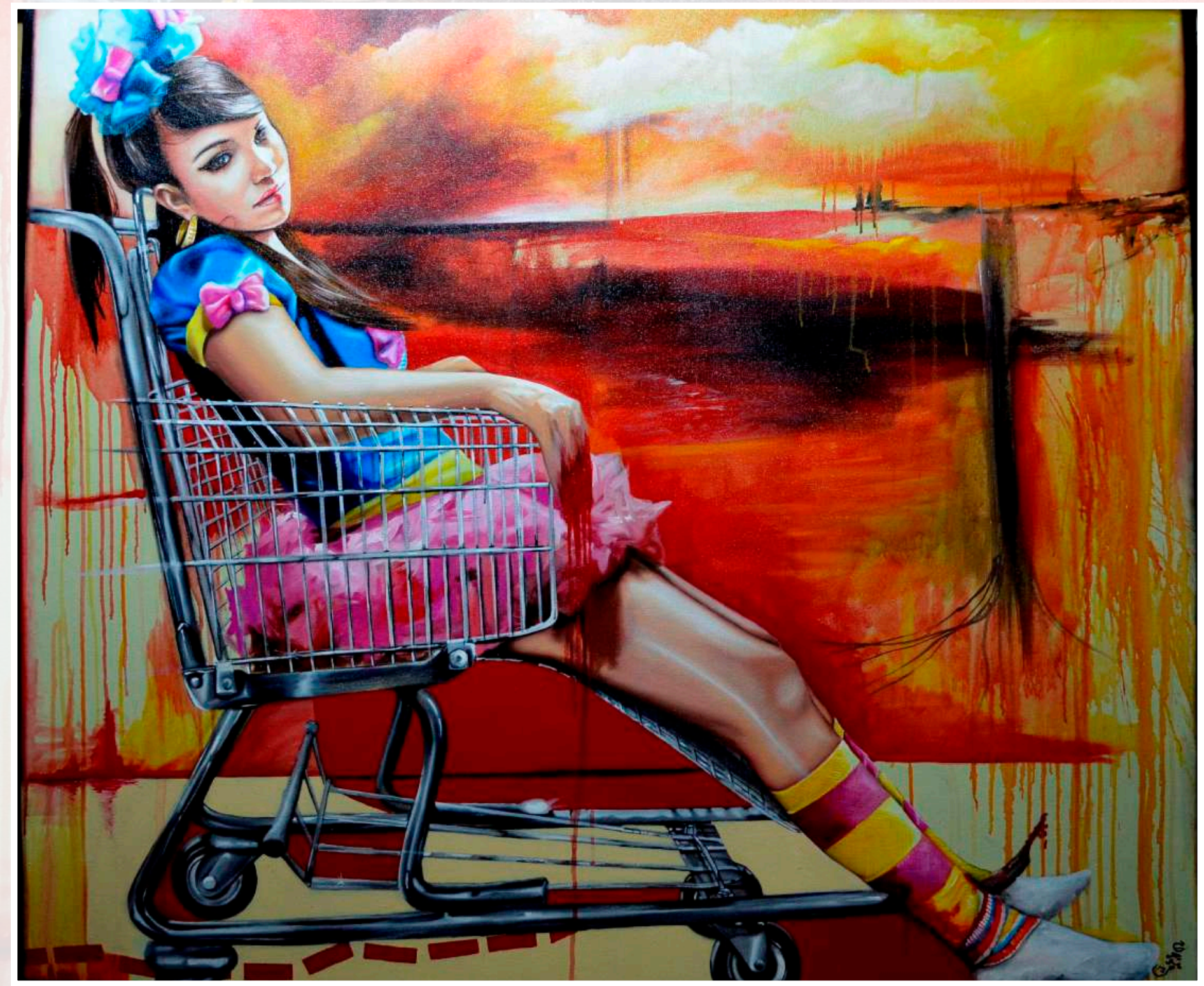

Como artista visual plasmo el retrato y figura humana realista, en conjunto con una simbología acorde a la temática de la obra. Me gusta ver mi obra como todo un proceso, el cual va desde la idea, el boceto, la elección del o la modelo, la toma de una fotografía o bien un dibujo, hasta la realización de la pintura. Generalmente utilizo el acrílico por la rapidez con la que puedo realizar obras en tonos vivos y cálidos. Pienso que en el discurso de la obra que realizo, trato hacer conciencia de lo caótico de la ciudad, del ser humano en sí y de la interacción del individuo con la naturaleza.

\section{- Carlos Robledo}

